\title{
BMJ Open Quality improvement collaborative aiming for Proactive HEAlthcare of Older People in Care Homes (PEACH): a realist evaluation protocol
}

\author{
Reena Devi, ${ }^{1,2}$ Julienne Meyer, ${ }^{3}$ Jay Banerjee, ${ }^{4,5}$ Claire Goodman, ${ }^{6}$ \\ John Raymond Fletcher Gladman, ${ }^{7}$ Tom Dening, ${ }^{8}$ Neil Chadborn, ${ }^{1}$ \\ Kathryn Hinsliff-Smith, ${ }^{1}$ Annabelle Long, ${ }^{1}$ Adeela Usman, ${ }^{1}$ Gemma Housley, ${ }^{9}$ \\ Clive Bowman, ${ }^{3}$ Finbarr Martin, ${ }^{10,11}$ Phillipa Logan, ${ }^{7}$ Sarah Lewis, ${ }^{12}$ \\ Adam Lee Gordon ${ }^{1,3}$
}

To cite: Devi R, Meyer J, Banerjee J, et al. Quality improvement collaborative aiming for Proactive HEAlthcare of Older People in Care Homes (PEACH): a realist evaluation protocol. BMJ Open 2018;8:e023287. doi:10.1136/ bmjopen-2018-023287

- Prepublication history for this paper is available online. To view these files, please visit the journal online (http://dx.doi. org/10.1136/bmjopen-2018023287).

Received 9 April 2018

Revised 5 July 2018

Accepted 24 July 2018
Check for updates

(C) Author(s) (or their employer(s)) 2018. Re-use permitted under CC BY-NC. No commercial re-use. See rights and permissions. Published by BMJ.

For numbered affiliations see end of article.

Correspondence to

Dr Reena Devi;

R.Devi@Leeds.ac.uk

\section{ABSTRACT}

Introduction This protocol describes a study of a quality improvement collaborative (QIC) to support implementation and delivery of comprehensive geriatric assessment (CGA) in UK care homes. The QIC will be formed of health and social care professionals working in and with care homes and will be supported by clinical, quality improvement and research specialists. QIC participants will receive quality improvement training using the Model for Improvement. An appreciative approach to working with care homes will be encouraged through facilitated shared learning events, quality improvement coaching and assistance with project evaluation.

Methods and analysis The QIC will be delivered across a range of partnering organisations which plan, deliver and evaluate health services for care home residents in four local areas of one geographical region. A realist evaluation framework will be used to develop a programme theory informing how QICs are thought to work, for whom and in what ways when used to implement and deliver CGA in care homes. Data collection will involve participant observations of the QIC over 18 months, and interviews/focus groups with QIC participants to iteratively define, refine, test or refute the programme theory. Two researchers will analyse field notes, and interview/focus group transcripts, coding data using inductive and deductive analysis. The key findings and linked programme theory will be summarised as contextmechanism-outcome configurations describing what needs to be in place to use QICs to implement service improvements in care homes.

Ethics and dissemination The study protocol was reviewed by the National Health Service Health Research Authority (London Bromley research ethics committee reference: 205840) and the University of Nottingham (reference: LT07092016) ethics committees. Both determined that the Proactive HEAlthcare of Older People in Care Homes study was a service and quality improvement initiative. Findings will be shared nationally and internationally through conference presentations, publication in peer-reviewed journals, a graphical illustration and a dissemination video.

\section{Strengths and limitations of this study}

- A realist evaluation approach will enable an in-depth study of how a quality improvement collaborative (QIC) intervention works (or not), for whom and also in what ways when used to implement and deliver comprehensive geriatric assessment (CGA) in care homes.

- Two researchers (RD and shared between NC, ALG, $\mathrm{JB}$, and $\mathrm{JM}$ ) will analyse the data, and the whole study team of multidisciplinary academics will be involved in interpreting the data during programme theory generation, testing and consolidation to improve validity of the findings.

- The study team will act as both intervention facilitators and evaluators, and thus will have first-hand experience of how a QIC approach works in this setting, for whom and in what ways.

- This dual role for the study team may introduce bias due to socially desirable responding, which will be mitigated through self-reflective techniques and member checking.

- This is a study of one QIC used to implement and deliver CGA in care homes in one region of the UK, and therefore, generalisability will pivot on establishing mid-range theory applicable in other settings.

\section{INTRODUCTION}

A large and growing number of older people with frailty live in care homes. The total care home population is approximately $433000,{ }^{1}$ of whom approximately 170000 have high dependency needs (needing 24-hour nursing care). ${ }^{2}$ The number with high dependency needs is forecast to increase to approximately 310000 by $2035 .^{2}$

This forecast presents challenges for health and social care providers. Current healthcare services do not adequately meet the needs of care home residents, let alone being 
prepared for future growth. Iliffe $e t a \grave{l}$ reported working relationships between the National Health Service (NHS) and care homes lack structure and purpose, with wide variation in the provision of both general and specialist healthcare services to care homes. The current challenge is to develop existing services to meet peoples' care needs. ${ }^{4}$ Comprehensive geriatric assessment (CGA) is the accepted standard process of caring for older people with frailty. The process starts with a holistic assessment of an older person, which is then used to develop a comprehensive care plan that is then delivered using multidisciplinary and coordinated specialist care. CGA has a strong evidence base showing improved outcomes, ${ }^{5}$ however, there is evidence that CGA is not currently taking place in care homes. ${ }^{6}$

One possible approach to facilitate the practice change necessary to implement CGA is a quality improvement collaborative (QIC) intervention. ${ }^{7}$ The QIC approach focuses on implementing evidence into practice by facilitating shared learning using 'Plan, Do, Study, Act' cycles to reflect and build on changes made in practice in an iterative way. In a QIC, clinical and quality improvement experts provide teams with guidance, improvement ideas, structured activities and encouragement to improve the quality of healthcare service, usually for a limited period of time (approximately 1-2 years). The way QICs are delivered vary, ${ }^{8}$ but they generally comprise five essential features: (1) focus is on a specified topic; (2) provision of ideas and support for improvement is provided by clinical and quality improvement experts; (3) participation by multiprofessional teams from multiple sites; (4) utilisation of a model for improvement (setting targets, collecting data and testing changes) and (5) the requirement for collaborative participants to engage in a series of structured activities. ${ }^{9}$

Recently, Wells et $a l^{10}$ systematically reviewed the evidence around QICs, and found that $83 \%$ of the 64 included studies reported an improvement in one or more of the study's primary effect measures. Four of the included studies were conducted in a care home setting and used the QIC intervention to improve care. The specific aims in these studies were to reduce falls, ${ }^{11}{ }^{12}$ reduce pressure ulcers ${ }^{13}$ and improve pain-management processes of care and outcomes. ${ }^{14}$ Three of these reported improved outcomes after the QIC, namely reduced pain prevalence,${ }^{14}$ reduced falls incidence, ${ }^{11}$ and improved pressure ulcer prevention and care. ${ }^{13}$

Systematic reviews, like the one conducted by Wells, are useful in describing the effectiveness of an intervention but cast less light on the mechanisms by which it operates. QICs are complex, multifaceted, context-sensitive, social and behavioural interventions. The intervention recruits collaborative teams of people employed across different organisations, environments and seniority levels. Effects of QIC interventions are likely to be a consequence of social interactions, and team dynamics which are influenced by organisational structures, cultures and social norms. It is important to understand these interactions,
Box 1 Initial programme theory-insights from literature describing generally how quality improvement collaboratives (QICS) are thought to work, and evidence describing barriers and enablers of using QIC interventions in the care home settings

\section{Insights from quality improvement literature describing how QICs are thought to work}

By collaborating and comparing practice, teams will be motivated to do things differently and make changes to practise that improve patient outcomes. $^{21}$

QICs are a learning organisation which empower teams to address quality problems through providing motivation, knowledge, skills and support. $^{22}$

The QIC intervention creates a collaborative environment which provides an opportunity for diverse participants to come together, reflect and learn. New learning and insights are then shared across the collaborative and taken back to employing organisations. ${ }^{23}$

\section{Barriers and enablers of using QIC interventions in care home settings}

Barriers:

- Fear of potential side effects when changing medication. ${ }^{14}$

- Problems occurring at the care home at the same time of the QI project (eg, staff shortages) resulted in the QI project being seen as a low priority. ${ }^{14}$

Difficulties applying quality improvement methodology. ${ }^{14}$

- High turnover of key members of staff involved in the QIC. ${ }^{14}$

- Complexity of the intervention and complexity of applying the change. ${ }^{12}$

Facilitators:

- One-on-one mentors kept quality improvement work as priority and simplified project tasks. ${ }^{14}$

- Providing simplified and incremental project steps to nursing home staff. ${ }^{14}$

- Effective communication (both inside and outside of formal meetings) to facilitate sharing and receiving new ideas, and passing on learning from pilot testing. ${ }^{11}$

- Allowing time for buy-in decision. ${ }^{11}$

- Engage wider range of care home staff in the process of quality improvement as multiple levels of staffing will affect the decision to adopt new care practices. ${ }^{12}$

- QIC project depended on self-selected, motivated and diligent nursing homes. ${ }^{13}$

and the different factors that influence the extent to which interventions are effective. ${ }^{915}$ Research literature describes how QIC interventions are generally thought to work, and describes barriers and enablers of using QIC interventions in care home settings. ${ }^{11-14}$ These insights are outlined in box 1, and comprise our initial programme theory.

This study will address a gap in the body of knowledge. The existing literature on care home QICs comes from outside the UK. It is possible that QICs will be challenged in this context in the UK because of the complex arrangement of health and social care provision in this setting, meaning that responsibilities can either be unclear or disputed. ${ }^{16}$ In addition, CGA is a particularly complex intervention, such that it may be less amenable 
to introduction by a QIC. Box 1 presents insights around how QICs are thought to work generally, and a description of the barriers and enablers of the QIC approach when used in care home settings. The current study will build on this and use a realist approach to develop a detailed programme theory presenting context-mechanism-outcome (CMO) configurations which answer the questions what works, for whom and in what ways when a QIC is used to improve delivery of CGA to people living in UK care homes.

\section{METHODS AND ANALYSIS \\ Study framework}

This study is one component of a programme of work called the Proactive HEAlthcare of Older People in Care Homes (PEACH) programme. The PEACH programme, funded by The Dunhill Medical Trust (grant number FOP1/0115), consists of four components. The first component, work-package one, is centred around delivering the QIC intervention, and carrying out a realist evaluation (described in this current protocol) to build a programme theory describing what works, for whom and in what ways when a QIC intervention is used to support delivery and implementation of CGA to people living in care homes. The second component uses a realist review methodology to review the evidence base and identify and characterise a programme theory that underpins the CGA intervention. ${ }^{17}$ The third and fourth work packages are orientated around evaluating the activity of the QIC, collecting data around healthcare service use and care home resident well-being. This article describes the protocol for work-package one.

QIC interventions involve complex human interactions. ${ }^{9}$ The ability of QICs to achieve the desired outcomes of service change and improvement are context sensitive and will change over time. We chose realist evaluation as an approach to understanding this because it focusses on describing how outcomes are achieved by mechanisms which operate to a greater or lesser extent depending on context. It looks for recurrent patterns of CMOs, described as demi-regularities. This enables a middle-range theory to be established which can be applied outside the context of study. The realist evaluation approach is theory driven and is based on realist philosophy. ${ }^{18}$ It addresses questions around how complex social, multifaceted interventions work and under what circumstances. One key tenet of the realist evaluation approach is that interventions have an implicit underlying theory which describes how change occurs. The findings will present a programme theory underpinned by CMO configurations describing the lessons learnt. The context (C) will describe the setting or conditions in which an intervention (in this case the QIC) is implemented. The mechanisms (M) will describe the causal forces, triggers or interactions that generate change within a given context. The outcomes $(\mathrm{O})$ will describe the actions or changes observed as a result of the QIC intervention. Each CMO configuration will present propositions stating what is it about the QIC intervention that makes it work, for whom and in what ways to implement and deliver CGA. ${ }^{18}$ This protocol follows the RAMESES standards for reporting realist evaluations. ${ }^{19}$

\section{Research aim}

Use a realist approach to establish the contextual and mechanistic factors associated with achieving desirable outcomes when a QIC intervention is used to improve the delivery of CGA to people living in care homes. A detailed programme theory will be developed that presents $\mathrm{CMO}$ configurations that describe the necessary contextual and mechanistic factors which generate desirable outcomes.

\section{Research question}

What works, for whom and in what ways when a QIC intervention is used to improve delivery of CGA to people living in UK care homes.

\section{The QIC intervention}

The aim of the QIC intervention will be to improve the delivery of CGA to people living in care homes.

The QIC intervention will be delivered by the project improvement team (ALG, JM, JB, and RD), a clinical academic geriatrician (ALG), a nurse leader with expertise in appreciative methods to promote quality of life in care homes (JM), a Health Foundation Quality Improvement Fellow (JB) and a health psychologist with interest in quality improvement $(\mathrm{RD})$.

The QIC intervention (September 2016 to February 2018) will involve four facilitated all-day shared learning events for the four multiprofessional groups of participants, at a neutral venue, where all participants will be away from their usual work environment. These events will comprise activities to build relationships between participants, training on CGA and quality improvement methodology, opportunities to reflect on local needs/ priorities, and sessions focused on developing quality improvement plans, updating groups on progress made, and sharing learning with each other. The activities and group exercises will use an appreciative inquiry approach that focuses on identifying what is working well and why, envisioning how things might be, identifying how to work together to make this happen and exploring what more needs to be done to make this happen more of the time. Each meeting will include opportunities to network with other groups located in differing geographical areas, working on the similar themed projects, and share similar quality improvement challenges.

In between the large-group learning sessions, participants will meet among themselves at their own site locations in order to review progress with their local quality improvement plan. Ongoing support from the project improvement team will be provided with coaching and mentoring, support with arranging meetings, email contact, directing teams to relevant local resources and relevant research, and support with evaluating the impact of their quality improvement plans. 


\section{Study setting}

This 24-month study (September 2016 to August 2018) will take place across four local areas of one geographical region within an area in the Midlands region of England, UK.

\section{Target population}

The aim of the QIC intervention is to improve the delivery of CGA into care homes in four local areas. In each of the four local areas a team of mixed multiprofessional health and social care professionals needed to deliver CGA will be recruited, and thus, a purposive sampling technique will be used. Depending on local staff availability and willingness to take part there will be approximately 7-10 participants in each of the four groups, with the following professional roles represented in each; general practitioners (GP), social care staff, nursing staff, therapists, geriatricians, voluntary sector staff, pharmacists, dementia specialists, care home workers/managers and members of the public. To recruit these participants the person with responsibilities of planning/purchasing healthcare services for older people in each of the four local areas will be asked to identify the relevant local key health and social care professionals to take part. Those who plan/ purchase healthcare services for older people will hold local knowledge and oversight of key and local health and social care staff members. Each group member will bring their expertise, experience and local knowledge to the quality improvement project. GPs and care home staff will be provided with backfill payment for their time taken to attend meetings as they are independent sector workers and only able to attend meetings if adequate staff cover is arranged to cover workload.

\section{Environment surrounding the evaluation}

The study will take place in a single region in the Midlands of England, UK. Care homes and NHS provider organisations within the geographical areas (four sites) will form the focus of interest. The NHS provider organisations are responsible for healthcare delivery for 670751 people, of which 73301 are over the age of 70 and commission primary healthcare delivery from 114 GP practices. The region includes a mixture of urban, suburban and rural settings, and a mixture of affluence and deprivation. The NHS provider organisations interface with two local authorities which commission statutory healthcare from 185 care homes. The variety of care provision within this region is such that findings from the area are therefore likely to be generalisable to most other UK settings. The local health and social care economies have previously been involved in a number of high-profile initiatives related to care homes and there is a track record of successful collaborative working in this regard.

\section{Data collection}

Observations, interviews and focus groups will be the core methods of data collection used to develop, test and consolidate the programme theory that will describe what works, for whom and in what ways when a QIC intervention is used to improve the delivery of CGA to care homes. These methods will enable a deeper insight and understanding into how participants interact with the QIC intervention, contextual influences, mechanistic factors and the outcomes produced. In particular, the interview and focus group methods will allow a deeper exploration into individual participant views, experiences and motivations while taking part in the QIC intervention. Interview and focus group methods will also allow the programme theory to be the subject matter and used to guide the conversation, while also enabling the researcher to ask open-ended questions to develop an understanding of the underlying particular contextual and mechanistic factors. Additional data describing the impact of the QIC will also be available, and will inform the $\mathrm{O}$ part of the $\mathrm{CMO}$ configurations in the programme theory.

\section{Observations}

These will include ethnographical observations of collaborative learning session meetings, smaller collaborative group meetings, and local CCG area steering group meetings. In particular, these observations will look at how members of the collaborative groups interact, engage and work together, and also the factors that seem to influence group working. During these meetings, groups will be observed, and field notes taken around the factors that appear to help the group to progress, and what more needs to be done to make it better.

Ethnographical observations of the local CCG site steering group meetings will provide insights into other care home-related activities taking place in the CCG area, and other information that may be relevant to the study. Observations made during the collaborative learning session meetings by the team will be reflected and discussed within PEACH study team meetings. Field notes will be recorded in all the meetings observed.

\section{Interviews/focus groups}

Interviews and focus groups will be carried out with the participants at each stage of theory development. These data will provide information about the QIC process and how it works. All interviews will be transcribed verbatim. Four groups of between 7 and 10 mixed multiprofessional health and social care professional participants will take part in the QIC intervention (described above). The aim is to interview each of these participants, giving a minimum sample size of approximately 28-40 participants. Additional theoretical sampling will be guided by emerging CMO frameworks and the need to inform these by insights from other professionals or care recipients with whom the collaborative have had contact. We anticipate conducting interviews/focus groups with participants more than once, and up to four times, during the course of the study, to gain an understanding of the intervention as it develops. 


\section{Additional data}

Field notes will be made from informal discussions with the participants, and from relevant documents such as meeting minutes and organisational reports. These will be examined to look for consistent patterns (referred to as demi-regularities) in the data and possible linkages which can then inform the development of the CMOs.

\section{Service-level outcome data}

Service-level outcomes describing hospital emergency admission rates, hospital length of stay, 30-day readmission rates, ambulance use, GP out of hours use for each care home will be available. These data measure the impact of the quality improvement work, and thus will help to interpret the outcomes of the quality improvement work carried out by the QIC participants. A monthly care home level breakdown will be available, and will provide an indication of how the QIC is affecting servicelevel use. These data will be collected using an automated algorithm designed to identify care home resident service use from NHS hospital databases. ${ }^{20}$ These data will be analysed using an interrupted time series approach.

\section{Qualitative data analysis}

Two researchers (RD and shared between NC, ALG, JB, and JM) will analyse field notes, and interview/focus group transcripts, coding data using both inductive and deductive analysis. Key themes describing important insights around contexts, mechanisms (resources and reasoning), and outcomes will be identified. Researchers will compare coding and any discrepancies in coding will be discussed, and where necessary other team members will be involved.

Coded data will be organised using NVivo software. Nodes will be created for every insight thought to be important for the programme theory describing how QICs work, for whom and in what ways when used to improve healthcare services delivered into care homes. Nodes will be organised into folders, and coded data imported to appropriate nodes. Each broad node will contain related data (relating to CMOs) and emerging patterns will be looked for in the data and CMOs assembled.

At the stages of programme theory generation, testing and consolidation, the whole study team will be involved in data interrogation and interpretation and will also be involved in ongoing discussions to verify interpretations and conclusions.

\section{Data synthesis}

The insights generated from observations, interviews/ focus groups, additional data (informal discussions with participants and relevant document analysis) and servicelevel outcomes data will be synthesised. A matrix will be generated, consisting of rows where each of the four sites will be represented and columns containing data on CMOs for each. Additional columns will contain servicelevel outcome data, and linkages in data will be made, helping to identify insights around how the QICs work and the impact on healthcare services. This matrix will help to synthesise the data, and thus enable the programme theory CMO configurations to be formed.

\section{Programme theory development}

Our study findings will present a programme theory that describes what works, for whom and in what ways when a QIC intervention is used to improve delivery of CGA in care homes. The stages of the realist enquiry will be: developing (stage 1), testing (stage 2) and consolidating (stage 3) the programme theory. This will be an iterative process, building a programme theory by collecting data from those taking part in QIC intervention activities. Data will be collected from the participants at every stage of theory development, and therefore, it is likely that participants may take part in repeat observations/interviews/focus groups at different stages of programme theory development. This is in line with Pawson and Tilley's ${ }^{18}$ recommendation that participant recruitment is based on 'CMO investigation potential'. Different participants will have different experiences and be able to reveal valuable insights around different parts of the underlying programme theory. Repeat observations, interviews and focus groups will be carried out with the same participants over the programme theory development stages.

\section{Stage 1: theory generation}

During stage 1, an initial rough programme theory will be developed. This will consist of initial CMO configurations generated from insights from relevant literature, interviews/focus groups and ethnographical observations of the collaborative groups during meetings. Data from these will be used to identify themes, categories, mechanisms and contextual factors associated with improved quality of healthcare. The relevant literature examined will include the broad quality improvement literature, broad QIC and QIC in care homes literature, and the communities of practice literature.

\section{Stage 2: theory testing and refinement}

The programme theory CMOs generated during stage 1 will be tested and interrogated through further observations of QIC meetings, and through conducting further interviews and focus groups. Participants taking part in stage 2 data collection will be selected purposively based on their 'CMO investigation potential'. ${ }^{18}$ The interviews and focus groups in this stage will take place towards the end of the QIC intervention. The programme theory CMOs will be the subject matter of these interviews and focus groups where the programme theory CMOs will be fed back and discussed with the participants. Once the CMOs are presented, the participants will be asked questions around whether or not the CMOs capture their experience, whether there is anything missing and if anything should be amended. The initial programme theory during this testing stage will either be accepted, rejected or modified. Refinement of the programme 
theory CMOs will be carried out iteratively and continuously tested in subsequent interviews and focus groups.

\section{Stage 3: theory consolidation and validation}

The refined programme theory will be reflected back to the participants during two consultation events. These will take place approximately 3 months after the QIC intervention completes. These will allow the participants to comment on the accuracy of the programme theory in mirroring their experiences of taking part in the QIC intervention. During this process, the programme theory CMOs will be presented to the participants and validation will be sought by checking if the participants agree or disagree. Modifications will be made in light of their comments. Other researcher groups known to the authors who have conducted similar projects will also be consulted in order to use their experience to help build and affirm the final programme theory.

\section{Public involvement}

Members of the public were involved in developing the overall PEACH programme: the research questions, aims/objectives, the study outcome measures and the study design. They read draft copies of the proposal, offered their comments, feedback, and ideas. These members of the public have first-hand experience of relatives in care homes. Their views, perspectives and priorities helped towards informing the overall PEACH programme study design.

The public will also be involved in the QIC intervention component of the PEACH programme which is described in this paper. Members of the public will be recruited to join the four groups taking part in the QIC intervention. Similar to the health and social care professionals, members of the public will take part in the QIC intervention activities. They will also form the study population from whom data will be collected throughout stages of programme theory generation, testing and consolidation.

Members of the public will also form part of an independent steering group committee who will regularly review the conduct of the study, study progress, emerging findings, and ensure validity and credibility.

\section{Ethics and dissemination}

The findings will be of interest to people planning and working on improving health and social care services delivered to care homes. The research community, including healthcare improvement scientists, will also be interested. The findings will be shared nationally and internationally through presenting at scientific conferences and publishing in a peer-reviewed journal. The findings will also be summarised creatively through a graphical illustration and a dissemination video, both of which will be shared nationally and internationally.

Two consultation events will also be held in local care homes taking part in the PEACH programme. The audience will comprise QIC intervention participants and the care home staff, and relatives of residents involved in the PEACH programme. At these events, other potential ways of disseminating the findings will be identified.

\section{Author affiliations}

${ }^{1}$ Division of Medical Sciences and Graduate Entry Medicine, School of Medicine, University of Nottingham, Nottingham, UK

${ }^{2}$ School of Healthcare, Faculty of Medicine and Health, University of Leeds, Leeds, UK

${ }^{3}$ School of Health Sciences, City University London, London, UK

${ }^{4}$ University Hospitals of Leicester NHS Trust, University of Leicester, Leicester, UK

${ }^{5}$ Department of Health Sciences, University of Leicester, Leicester, UK

${ }^{6}$ Centre for Research in Primary and Community Care, University of Hertfordshire, Hatfield, UK

${ }^{7}$ Division of Rehabilitation and Ageing, School of Medicine, University of Nottingham, Nottingham, UK

${ }^{8}$ Division of Psychiatry and Applied Psychology, School of Medicine, University of Nottingham, Nottingham, UK

${ }^{9}$ Health Analytics and Informatics, East Midlands Academic Health Science Network, Nottingham, Nottingham, UK

${ }^{10}$ Ageing and Health, Guy's and St Thomas' NHS Foundation Trust, London, UK

${ }^{11}$ Population Health Sciences, King's College London, London, UK

${ }^{12}$ Division of Epidemiology and Public Health, School of Medicine, University of Nottingham, Nottingham, UK

Acknowledgements QIC meetings will be funded by the East Midlands Academic Health Sciences Network Patient Safety Collaborative as part of their programme of dissemination of gold standard practice across the East Midlands. We would also like to acknowledge the contribution members of the public made to the overall PEACH programme, and to the QIC aspect of the project that is described in this paper.

Contributors ALG is the principal investigator of the PEACH project. ALG, JM, JB and $\mathrm{RD}$ delivered the QIC intervention. $\mathrm{RD}$ is main researcher carrying out data collection and data analysis. NC, ALG, JM, and JB are also carrying out data coding and analysis tasks. KH-S, AL, AU and GH are members of the research team, with CG, JRFG, TD, CB, PL, SL and FM who are coinvestigators of the PEACH programme. All authors contributed to the writing/editing of this protocol, and read and approved the final manuscript.

Funding The PEACH programme is funded by The Dunhill Medical Trust (grant number FOP1/0115).

Competing interests None declared.

Patient consent Not required.

Ethics approval Both the National Health Service and University of Nottingham research ethics committees considered this study to be service development project and thus full ethics approval was not required (London Bromley REC ref: 205840, University of Nottingham ref: LT07092016).

Provenance and peer review Not commissioned; externally peer reviewed.

Open access This is an open access article distributed in accordance with the Creative Commons Attribution Non Commercial (CC BY-NC 4.0) license, which permits others to distribute, remix, adapt, build upon this work non-commercially, and license their derivative works on different terms, provided the original work is properly cited, appropriate credit is given, any changes made indicated, and the use is non-commercial. See: http://creativecommons.org/licenses/by-nc/4.0/.

\section{REFERENCES}

1. Buisson L. Care of older people. 27th edn, 2015

2. Kingston $A$, Wohland $P$, Wittenberg $R$, et al. Is late-life dependency increasing or not? A comparison of the Cognitive Function and Ageing Studies (CFAS). The Lancet 2017;390:1676-84.

3. Iliffe S, Davies SL, Gordon AL, et al. Provision of NHS generalist and specialist services to care homes in England: review of surveys. Prim Health Care Res Dev 2016;17:122-37.

4. Gordon AL, Goodman C, Davies SL, et al. Optimal healthcare delivery to care homes in the UK: a realist evaluation of what supports effective working to improve healthcare outcomes. Age Ageing 2018:595-603. 
5. Ellis G, Whitehead MA, Robinson D, et al. Comprehensive geriatric assessment for older adults admitted to hospital: meta-analysis of randomised controlled trials. BMJ 2011;343:d6553.

6. Goodman C. Dying in care homes when advance care planning requires wraparound care. Palliative Medicine 2018;32:312.

7. Kilo CM. A framework for collaborative improvement: lessons from the Institute for Healthcare Improvement's Breakthrough Series. Qual Manag Health Care 1998;6:1-13.

8. Nadeem E, Olin SS, Hill LC, et al. Understanding the components of quality improvement collaboratives: a systematic literature review. Milbank Q 2013;91:354-94.

9. Schouten LM, Hulscher ME, van Everdingen JJ, et al. Evidence for the impact of quality improvement collaboratives: systematic review. BMJ 2008;336:1491-4.

10. Wells S, Tamir O, Gray J, et al. Are quality improvement collaboratives effective? A systematic review. BMJ Qual Saf 2018;27:226-40.

11. Arling PA, Abrahamson $\mathrm{K}$, Miech EJ, et al. Communication and effectiveness in a US nursing home quality-improvement collaborative. Nurs Health Sci 2014;16:291-7.

12. Colón-Emeric C, Schenck A, Gorospe J, et al. Translating evidencebased falls prevention into clinical practice in nursing facilities: Results and lessons from a quality improvement collaborative. J Am Geriatr Soc 2006;54:1414-8.

13. Lynn J, West J, Hausmann S, et al. Collaborative clinical quality improvement for pressure ulcers in nursing homes. J Am Geriatr Soc 2007;55:1663-9.
14. Baier RR, Gifford DR, Patry G, et al. Ameliorating pain in nursing homes: a collaborative quality-improvement project. J Am Geriatr Soc 2004;52:1988-95.

15. Lindenauer PK. Effects of quality improvement collaboratives. BMJ 2008;336:1448-9.

16. Robbins I, Gordon A, Dyas J, et al. Explaining the barriers to and tensions in delivering effective healthcare in UK care homes: a qualitative study. BMJ Open 2013;3:e003178.

17. Zubair M, Chadborn NH, Gladman JRF, et al. Using comprehensive geriatric assessment for quality improvements in healthcare of older people in UK care homes: protocol for realist review within proactive healthcare of older people in care homes (PEACH) study. BMJ Open 2017;7:e017270

18. Pawson R, Tilley N. Realistic evaluation. California: sage, 1997.

19. Wong G, Westhorp G, Manzano A, et al. Rameses ii reporting standards for realist evaluations. BMC Med 2016;14:96.

20. Housley G, Lewis S, Usman A, et al. Accurate identification of hospital admissions from care homes; development and validation of an automated algorithm. Age Ageing 2018;47:387-91.

21. de SilvaD. No 21 Improvement collaboratives in health care. The Health Foundation Inspiring Improvement ed. London: The Health Foundation, 2014.

22. ØVretveit J, Bate P, Cleary P, et al. Quality collaboratives: lessons from research. Qual Saf Health Care 2002;11:345-51.

23. Shaw EK, Chase SM, Howard J, et al. More black box to explore: how quality improvement collaboratives shape practice change. $J$ Am Board Fam Med 2012;25:149-57. 